\title{
$O$-Nucleophilic Amino-Alcohol Acyl-Transfer Catalysts: the Effect of Acidity of the Hydroxyl Group on the Activity of the Catalyst
}

\author{
Kjirsten A. Wayman and Tarek Sammakia* \\ Department of Chemistry and Biochemistry, University of Colorado \\ Boulder, Colorado 80309-0215
}

Compounds 14, 16, 17, and 18 were prepared via 4-(dimethylamino)-6-methyl-pyridine-2carbaldehyde, which was prepared from 4-DMAP, and compound 15 was prepared from 2-bromo6-methyl-4-(dimethylamino)-pyridine as shown below.
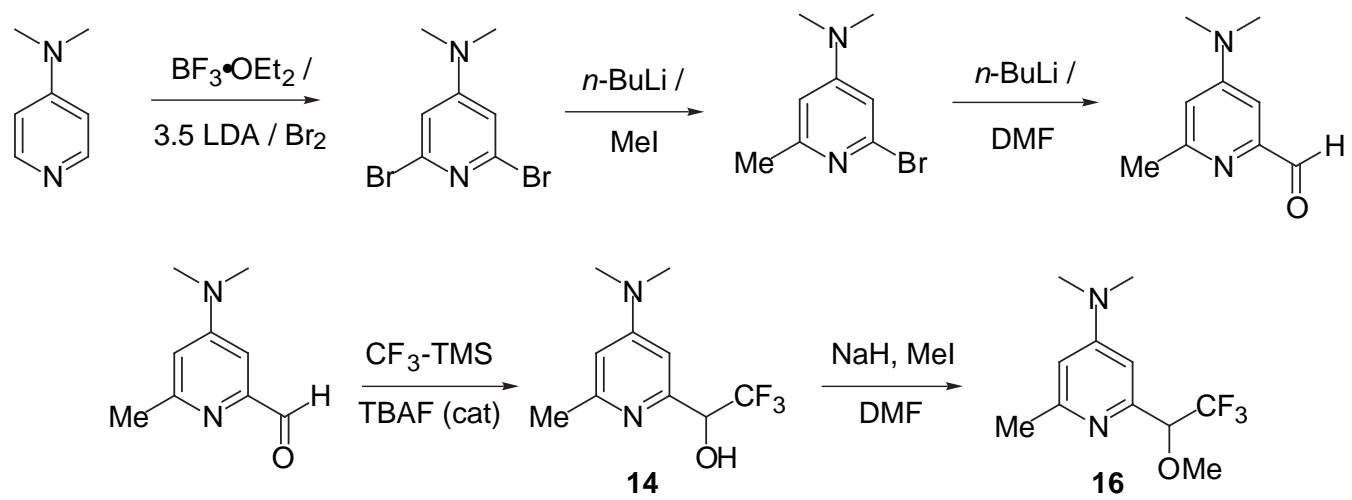<smiles>Cc1cc(N(C)C)cc(C(=O)c2cc(Br)cc(C(O)c3c(F)c(F)c(F)c(C(O)c4cc(N(C)C)cc(N(C)C)n4)c3F)n2)n1</smiles><smiles>Cc1cc(N(C)C)cc(Br)n1</smiles>
below.

Compounds 20 - 23 were prepared from 2-Pyrrolidin-1-ylmethyl-benzaldehyde ${ }^{1}$ as shown 


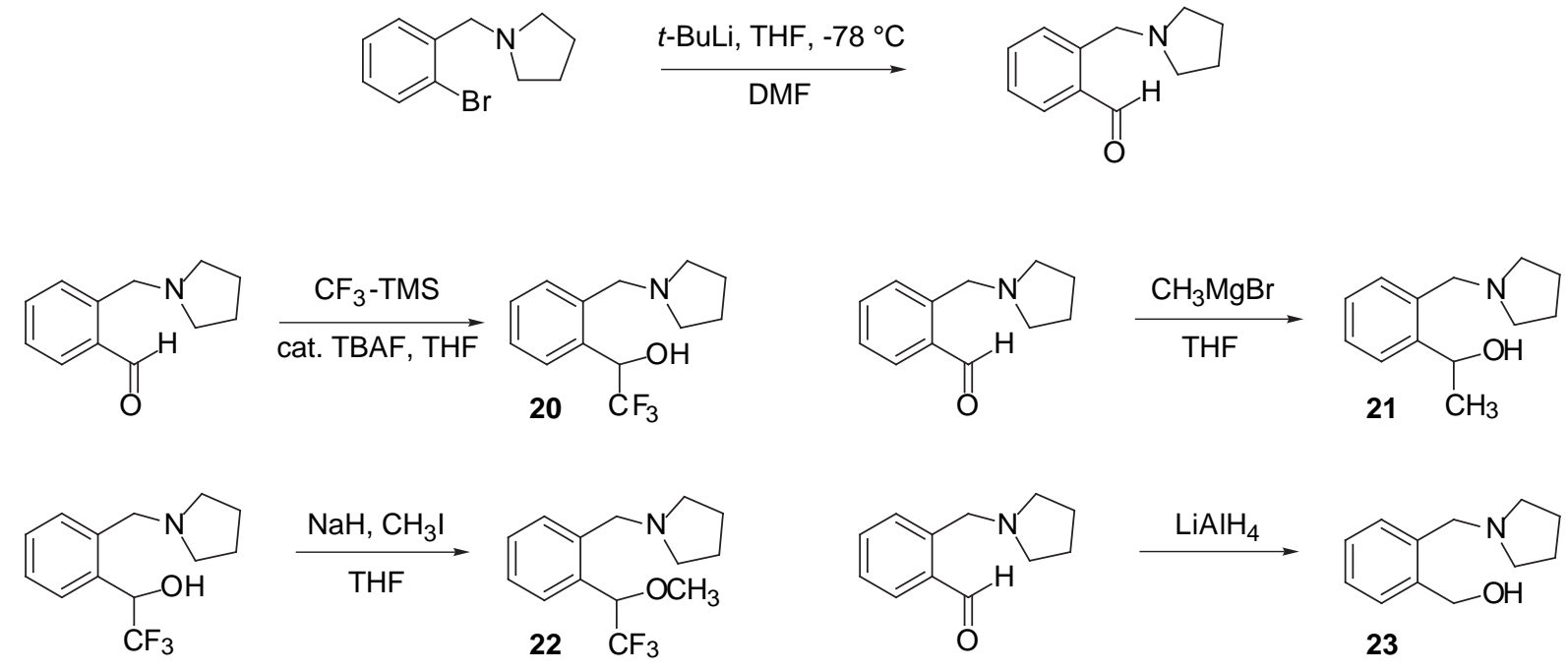

\section{General experimental procedures:}

All air or moisture sensitive reactions were performed in oven-dried glassware under a nitrogen atmosphere using solvents purified according to standard procedures. ${ }^{2}{ }^{1} \mathrm{H}$ NMR spectra were obtained at either $300 \mathrm{MHz}$ or $500 \mathrm{MHz},{ }^{13} \mathrm{C}$ NMR spectra were obtained at either $75 \mathrm{MHz}$ or $125 \mathrm{MHz}$ with ${ }^{1} \mathrm{H}$ decoupling (WALTZ), and ${ }^{19} \mathrm{~F}$ spectra were obtained at either $376 \mathrm{MHz}$, or $470 \mathrm{MHz}$. All NMR data was obtained in chloroform- $d$ as solvent. Chemical shifts are reported in ppm referenced to residual chloroform at $7.24 \mathrm{ppm}$ for ${ }^{1} \mathrm{H}$ NMR and $77.0 \mathrm{ppm}$ for ${ }^{13} \mathrm{C}$ NMR. ${ }^{19} \mathrm{~F}$ NMR chemical shifts are reported relative to $\mathrm{CFCl}_{3}$, set at $0.0 \mathrm{ppm}$. Infrared spectra were recorded as thin films on sodium chloride plates.

\section{Kinetics of ethanolysis of $p$-Nitrophenyl Methoxyacetate:}

Kinetic experiments for the methanolysis of $p$-nitrophenyl methoxyacetate were monitored by ${ }^{1} \mathrm{H}$ NMR at $500 \mathrm{MHz}$ using a Varian Inova-500 spectrometer. Chloroform- $d$ was freshly distilled from calcium hydride immediately prior to each experiment. Samples were prepared from stock solutions of the ester and the catalyst, neat methanol- $d_{4}$, and neat $p$-methylanisole (as a standard). The chloroform- $d$ solutions were $0.1 \mathrm{M}$ in ester, $1.0 \mathrm{M}$ in methanol, and $0.01 \mathrm{M}$ in catalyst at a total volume of $750 \mu \mathrm{L}$, plus $3 \mu \mathrm{L}$ of standard. Reactants were combined directly into NMR tubes. Reactions were run at $20 \pm 2{ }^{\circ} \mathrm{C}$. An NMR spectrum was taken before the catalyst was added, and reaction progress was monitored by the disappearance of aromatic resonances of $p$ nitrophenyl methoxyacetate and the appearance of methylene protons of methyl- $d_{3}$ methoxyacetate. The state of the catalyst was monitored by the appearance and disappearance of the proton geminal to the hydroxy or acyloxy group in the unacylated and acylated catalyst. Percent compound was plotted as a function of time to analyze the pseudo-zero order reactions. The rates were calculated from the steady-state slopes corresponding to the growth of methyl- $d_{3}$ methoxyacetate. Reactions displayed clean conversion with no detectable formation of side products.

\section{2,6-Dibromo-4-(dimethylamino)-pyridine}<smiles>CN(C)c1cc(Br)nc(Br)c1</smiles>

Boron trifluoride diethyl etherate $(22.8 \mathrm{~mL}, 180 \mathrm{mmol}, 1.1$ equiv) was added via cannula to a solution of 4-dimethylaminopyridine (20.0 g, $164 \mathrm{mmol}, 1.0$ equiv) in tetrahydrofuran $(700 \mathrm{~mL})$ 
at $0{ }^{\circ} \mathrm{C}$. The resulting suspension was allowed to stir at $0{ }^{\circ} \mathrm{C}$ for 30 minutes then cooled to $-78{ }^{\circ} \mathrm{C}$. In a separate flask, $n$-butyllithium $(1.6 \mathrm{M}$ in hexanes, $256 \mathrm{~mL}, 410 \mathrm{mmol}, 2.5$ equiv) was added via cannula to a $0{ }^{\circ} \mathrm{C}$ solution of diisopropyl amine $(53.7 \mathrm{~mL}, 410 \mathrm{mmol}, 2.5$ equiv) in tetrahydrofuran $(300 \mathrm{~mL})$ and allowed to stir 30 minutes. The resulting lithium diisopropyl amide solution was then added via cannula to the $-78{ }^{\circ} \mathrm{C}$ solution of the pyridine boron trifluoride complex. The mixture was stirred for 25 minutes after the addition was complete. Bromine $(25 \mathrm{~mL}, 492 \mathrm{mmol}$, 3.0 equiv) was then added and the mixture was warmed to room temperature and allowed to stir overnight. Saturated sodium thiosulfate was added and the layers were separated. The aqueous phase was extracted with ethyl acetate $(3 \mathrm{x})$ and the combined organic layers were washed with brine and dried over $\mathrm{MgSO}_{4}$. After removing the solvents under reduced pressure, the residue was purified by flash chromatography (2:1 hexanes/ethyl acetate) followed by recrystallization (hexanes/methylene chloride) to provide $10.5 \mathrm{~g} \mathrm{(23 \% )}$ of 2,6-dibromo-4-(dimethylamino)-pyridine as a light pink crystalline solid: m.p. $148{ }^{\circ} \mathrm{C} .{ }^{\mathrm{H}} \mathrm{H} \mathrm{NMR}\left(500 \mathrm{MHz}, \mathrm{CDCl}_{3}\right): \delta 6.58(\mathrm{~s}, 2 \mathrm{H}), 2.97(\mathrm{~s}$, $6 \mathrm{H}) .{ }^{13} \mathrm{C} \mathrm{NMR}\left(125 \mathrm{MHz}, \mathrm{CDCl}_{3}\right): \delta 156.64,140.77,108.92,51.71,39.46$. IR 1586, 966, 811 $\mathrm{cm}^{-1}$. TLC $\mathrm{R}_{\mathrm{f}}=0.48\left(2: 1\right.$ hexanes/ethyl acetate). Anal Calcd for $\mathrm{C}_{7} \mathrm{H}_{8} \mathrm{~N}_{2} \mathrm{Br}_{2}: \mathrm{C}, 30.03 ; \mathrm{H}, 2.88 ; \mathrm{N}$, 10.01. Found: C, 29.87; H, 2.80; N, 9.91.

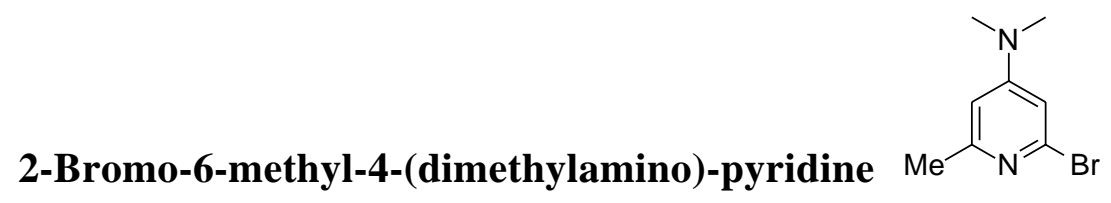

$n$-Butyllithium (1.6 M in hexanes, $8.4 \mathrm{~mL}, 13.4 \mathrm{mmol}, 1.1$ equiv) was added dropwise via cannula to a solution of 2,6-dibromo-4-(dimethylamino)-pyridine (3.42 g, $12.2 \mathrm{mmol}, 1.0$ equiv) in tetrahydrofuran $(125 \mathrm{~mL})$ at $-78^{\circ} \mathrm{C}$. The mixture was warmed to $-45^{\circ} \mathrm{C}$ for 15 minutes, and then cooled again to $-78^{\circ} \mathrm{C}$. Methyl iodide $(1.17 \mathrm{~mL}, 18.3 \mathrm{mmol}, 1.5$ equiv) was added to the mixture via syringe and then warmed to $-45^{\circ} \mathrm{C}$ for 2 hours. After warming to room temperature, saturated ammonium chloride $(100 \mathrm{~mL})$ was added to the reaction mixture and the layers were separated. The aqueous layer was extracted twice with ethyl acetate. The combined organic layers were dried over $\mathrm{MgSO}_{4}$ and concentrated to a yellow oil. Flash chromatography (2:1 hexanes/ethyl acetate) provided $2.03 \mathrm{~g}(77 \%)$ of 2-bromo-6-methyl-4-(dimethylamino)-pyridine: m.p. $49-51{ }^{\circ} \mathrm{C}$. ${ }^{1} \mathrm{H}$ NMR $\left(500 \mathrm{MHz}, \mathrm{CDCl}_{3}\right): \delta 6.46(\mathrm{~d}, J=2.2 \mathrm{~Hz}, 1 \mathrm{H}), 6.26(\mathrm{~d}, J=2.2 \mathrm{~Hz}, 1 \mathrm{H}), 2.94(\mathrm{~s}, 6 \mathrm{H}), 2.37$ $(\mathrm{s}, 3 \mathrm{H}) .{ }^{13} \mathrm{C}$ NMR $\left(125 \mathrm{MHz}, \mathrm{CDCl}_{3}\right): \delta 158.71,156.12,142.26,106.75,105.09,39.25,24.50$. IR $1600,1526,1131 \mathrm{~cm}^{-1}$. TLC $\mathrm{R}_{\mathrm{f}}=0.38$ (2:1 hexanes/ethyl acetate). Anal Calcd for $\mathrm{C}_{8} \mathrm{H}_{11} \mathrm{~N}_{2} \mathrm{Br}: \mathrm{C}$, 44.67; H, 5.15; N, 13.02. Found: C, 44.87; H, 4.96; N, 12.98.

\section{4-(Dimethylamino)-6-methyl-pyridine-2-carbaldehyde}

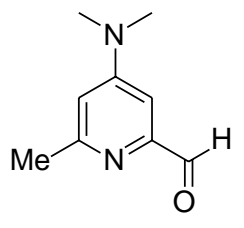

$n$-Butyllithium (1.6 M in hexanes, $4.79 \mathrm{~mL}, 7.67 \mathrm{mmol}, 1.1$ equiv) was added via cannula to a solution of 2-bromo-6-methyl-4-(dimethylamino)-pyridine (1.5 g, $6.97 \mathrm{mmol}, 1.0$ equiv) in tetrahydrofuran $(70 \mathrm{~mL})$ at $-78^{\circ} \mathrm{C}$. The mixture was allowed to stir for 45 minutes at $-78{ }^{\circ} \mathrm{C}$. Distilled $N, N$-dimethylformamide $(810 \mu \mathrm{L}, 10.5 \mathrm{mmol}, 1.5$ equiv) was added to the mixture via syringe and then warmed to room temperature. Saturated sodium carbonate $(50 \mathrm{~mL})$ was added and the mixture was extracted three times with ethyl acetate. The combined organic layers were dried over $\mathrm{MgSO}_{4}$ and concentrated. Flash chromatography (2:1 hexanes/ethyl acetate) provided $1.05 \mathrm{~g} \mathrm{(92 \% )}$ of 4-(dimethylamino)-6-methyl-pyridine-2-carbaldehyde as a pale yellow solid: m.p. $76-77^{\circ} \mathrm{C} .{ }^{1} \mathrm{H}$ NMR $\left(500 \mathrm{MHz}, \mathrm{CDCl}_{3}\right): \delta 9.91(\mathrm{~s}, 1 \mathrm{H}), 7.00(\mathrm{~d}, J=2.2 \mathrm{~Hz}, 1 \mathrm{H}), 6.48(\mathrm{~d}, J=2.4$ $\mathrm{Hz}, 1 \mathrm{H}), 3.00(\mathrm{~s}, 6 \mathrm{H}), 2.49(\mathrm{~s}, 3 \mathrm{H}) .{ }^{13} \mathrm{C} \mathrm{NMR}\left(125 \mathrm{MHz}, \mathrm{CDCl}_{3}\right): \delta 194.77,158.93,155.14$, $152.75,109.05,102.49,51.70,39.23,24.49$. TLC $\mathrm{R}_{\mathrm{f}}=0.13$ (2:1 hexanes/ethyl acetate). Anal 
Calcd for $\mathrm{C}_{9} \mathrm{H}_{12} \mathrm{~N}_{2} \mathrm{O}$ : C, 65.83; H, 7.37; N, 17.06. Found: C, 65.71; H, 7.25; N, 16.96.

\section{1-(4-Dimethylamino-6-methyl-pyridin-2-yl)-2,2,2-trifluoro-ethanol (14)}

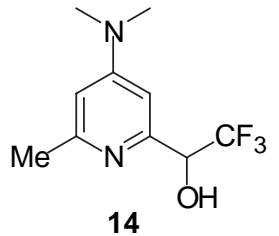

Tetrabutylammonium fluoride hydrate $(1 \mathrm{M}$ in tetrahydrofuran, $0.5 \mathrm{~mL})$ was added via cannula to a $0{ }^{\circ} \mathrm{C}$ solution of 4-(dimethylamino)-6-methyl-pyridine-2-carbaldehyde ( $200 \mathrm{mg}, 1.22$ mmol, 1.0 equiv) and trimethyl(trifluoromethyl)silane $(0.5 \mathrm{M}$ in tetrahydrofuran, $2.92 \mathrm{~mL}, 1.46$ mmol, 1.2 equiv) in tetrahydrofuran $(3 \mathrm{~mL})$. The solution was allowed to warm to room temperature and allowed to stir overnight. Hydrochloric acid (6 M, $400 \mu \mathrm{L}, 2.44 \mathrm{mmol}, 2.0$ equiv) was added and the mixture was allowed to stir for 1 hour. Excess solid sodium carbonate was then added to neutralize the hydrochloric acid. The resulting mixture was diluted with ethyl acetate, filtered over $\mathrm{MgSO}_{4}$ and concentrated under reduced pressure. Flash chromatography (ethyl acetate) provided $185 \mathrm{mg}(65 \%)$ of 14 as a white solid: m.p. $124-125{ }^{\circ} \mathrm{C} .{ }^{1} \mathrm{H} \mathrm{NMR}(500 \mathrm{MHz}$, $\left.\mathrm{CDCl}_{3}\right): \delta 6.36(\mathrm{~s}, 2 \mathrm{H}), 6.10(\mathrm{br}, 1 \mathrm{H}), 4.82\left(\mathrm{q}, J_{\mathrm{H}-\mathrm{F}}=6.9 \mathrm{~Hz}, 1 \mathrm{H}\right), 3.00(\mathrm{~s}, 6 \mathrm{H}), 2.43(\mathrm{~s}, 3 \mathrm{H}) .{ }^{13} \mathrm{C}$ $\operatorname{NMR}\left(125 \mathrm{MHz}, \mathrm{CDCl}_{3}\right): \delta 156.71,155.46,150.18,124.38\left(\mathrm{q}, J_{\mathrm{C}-\mathrm{F}}=283 \mathrm{~Hz}\right), 106.19,102.12$, $70.30\left(\mathrm{q}, J_{\mathrm{C}-\mathrm{F}}=31.7 \mathrm{~Hz}\right), 39.26,24.27 .{ }^{19} \mathrm{~F} \mathrm{NMR}\left(376 \mathrm{MHz}, \mathrm{CDCl}_{3}\right): \delta-78.49\left(\mathrm{~d}, J_{\mathrm{H}-\mathrm{F}}=6.0 \mathrm{~Hz}\right)$. IR $3000-3150,1610,1161,1119 \mathrm{~cm}^{-1}$. TLC R $\mathrm{R}_{\mathrm{f}}=0.29$ (2:1 hexanes/ethyl acetate). Anal Calcd for $\mathrm{C}_{10} \mathrm{H}_{13} \mathrm{~F}_{3} \mathrm{~N}_{2} \mathrm{O}: \mathrm{C}, 51.28 ; \mathrm{H}, 5.59 ; \mathrm{N}, 11.96$. Found: $\mathrm{C}, 51.12 ; \mathrm{H}, 5.48 ; \mathrm{N}, 11.89$.

\section{1-(4-Dimethylamino-6-methyl-pyridin-2-yl)-ethanol (15)}

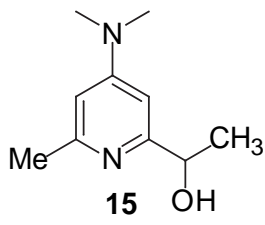

$n$-Butyllithium ( $1.6 \mathrm{M}$ in hexanes, $320 \mu \mathrm{L}, 0.51 \mathrm{mmol}, 1.1$ equiv) was added via syringe to a solution of 2-bromo-6-methyl-4-(dimethylamino)-pyridine (100 mg, $0.465 \mathrm{mmol}, 1.0$ equiv) in tetrahydrofuran $(5 \mathrm{~mL})$ at $-78{ }^{\circ} \mathrm{C}$. The mixture was allowed to stir for 45 minutes at $-78{ }^{\circ} \mathrm{C}$. Distilled acetaldehyde ( $130 \mu \mathrm{L}, 2.33 \mathrm{mmol}, 5.0$ equiv) was added to the mixture via syringe and then warmed to room temperature. Saturated sodium carbonate $(5 \mathrm{~mL})$ was added and the mixture was extracted three times with ethyl acetate. The combined organic layers were dried over $\mathrm{MgSO}_{4}$ and concentrated to a white solid. Flash chromatography using a gradient elution (ethyl acetate/methanol) provided $51 \mathrm{mg}(61 \%)$ of $15 .{ }^{1} \mathrm{H}$ NMR $\left(500 \mathrm{MHz}, \mathrm{CDCl}_{3}\right): \delta 6.26(\mathrm{~d}, J=2.2$ $\mathrm{Hz}, 1 \mathrm{H}), 6.24(\mathrm{~d}, J=2.4 \mathrm{~Hz}, 1 \mathrm{H}), 4.71(\mathrm{q}, J=6.6 \mathrm{~Hz}, 1 \mathrm{H}), 2.98(\mathrm{~s}, 6 \mathrm{H}), 2.42(\mathrm{~s}, 3 \mathrm{H}), 1.45(\mathrm{~d}, J=$ $6.4 \mathrm{~Hz}, 3 \mathrm{H}) .{ }^{13} \mathrm{C}$ NMR $\left(125 \mathrm{MHz}, \mathrm{CDCl}_{3}\right): \delta 162.46,156.59,155.61,104.60,99.34,68.50$, $39.29,24.45,24.42$. IR $3100-3500,1608,1548,1509,1117,1018 \mathrm{~cm}^{-1}$. TLC $\mathrm{R}_{\mathrm{f}}=0.17$ (methanol). Anal Calcd for $\mathrm{C}_{10} \mathrm{H}_{16} \mathrm{~N}_{2} \mathrm{O}: \mathrm{C}, 66.64 ; \mathrm{H}, 8.95 ; \mathrm{N}, 15.54$. Found: $\mathrm{C}, 67.02 ; \mathrm{H}, 8.84 ; \mathrm{N}$, 15.30 .

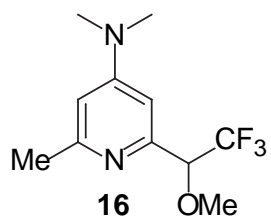

\section{Dimethyl-[2-methyl-6-(2,2,2-trifluoro-1-methoxy-ethyl)-pyridin-4-yl]-amine (16)}

A solution of 1-(4-dimethylamino-6-methyl-pyridin-2-yl)-2,2,2-trifluoro-ethanol (30.5 mg, 
$0.130 \mathrm{mmol}, 1.0$ equiv) in $N, N$-dimethylformamide $(1 \mathrm{~mL})$ was added via cannula to a suspension of sodium hydride (16 mg, $0.667 \mathrm{mmol}, 5$ equiv) in $N, N$-dimethylformamide $(0.5 \mathrm{~mL})$. The mixture turned orange after stirring 5 minutes. Methyl iodide was added via syringe to the alkoxide solution and then the reaction was allowed to stir overnight. The $N, N$-dimethylformamide was removed under reduced pressure. The remaining residue was dissolved in chloroform, washed with water, dried over $\mathrm{MgSO}_{4}$ and concentrated. Purification by flash chromatography using a gradient elution (5:1 hexanes/ethyl acetate, 2:1 hexanes/ethyl acetate, then ethyl acetate) provided $13 \mathrm{mg}$ $(41 \%)$ of $16 .{ }^{1} \mathrm{H}$ NMR $\left(500 \mathrm{MHz}, \mathrm{CDCl}_{3}\right): \delta 6.58(\mathrm{~d}, J=2.0 \mathrm{~Hz}, 1 \mathrm{H}), 6.35(\mathrm{~d}, J=2.4 \mathrm{~Hz}, 1 \mathrm{H})$, $4.56\left(\mathrm{q}, J_{\mathrm{H}-\mathrm{F}}=6.6 \mathrm{~Hz}, 1 \mathrm{H}\right), 3.45(\mathrm{~s}, 3 \mathrm{H}), 2.99(\mathrm{~s}, 6 \mathrm{H}), 2.43(\mathrm{~s}, 3 \mathrm{H}) .{ }^{13} \mathrm{C}$ NMR $\left(125 \mathrm{MHz}, \mathrm{CDCl}_{3}\right)$ : $\delta 157.88,155.38,152.43,123.74\left(\mathrm{q}, J_{\mathrm{C}-\mathrm{F}}=282 \mathrm{~Hz}\right), 106.13,102.24,83.11\left(\mathrm{q}, J_{\mathrm{C}-\mathrm{F}}=30.0 \mathrm{~Hz}\right)$, 58.48, 39.22, 24.64. ${ }^{19} \mathrm{~F} \mathrm{NMR}\left(376 \mathrm{MHz} \mathrm{CDCl}_{3}\right): \delta-76.36\left(\mathrm{~d}, J_{\mathrm{H}-\mathrm{F}}=9.0 \mathrm{~Hz}\right) . \quad \mathrm{IR} 1615,1548$, $1511,1271,1163,1133 \mathrm{~cm}^{-1}$. TLC $\mathrm{R}_{\mathrm{f}}=0.47$ (ethyl acetate). Anal Calcd for $\mathrm{C}_{11} \mathrm{H}_{15} \mathrm{~F}_{3} \mathrm{~N}_{2} \mathrm{O}: \mathrm{C}$, 53.22; H, 6.09; N, 11.28. Found: C, 53.05; H, 5.98; N, 10.88 .

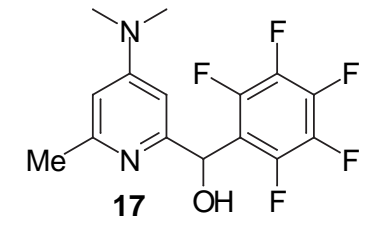

\section{(4-Dimethylamino-6-methyl-pyridin-2-yl)-pentafluorophenyl-methanol (17)}

Bromopentafluorobenzene $(83.5 \mu \mathrm{L}, 0.670 \mathrm{mmol}, 1.1$ equiv) was added via syringe to a flask containing magnesium turnings $(22.0 \mathrm{mg}, 0.914 \mathrm{mmol}, 1.5$ equiv) and diethyl ether $(0.5 \mathrm{~mL})$. The mixture was sonicated for 5 minutes at which point it appeared dark brown, and then diluted with diethyl ether $(2 \mathrm{~mL})$. The resulting Grignard reagent was then cannulated into a flask containing a solution of 4-(dimethylamino)-6-methyl-pyridine-2-carbaldehyde (100 mg, 0.609 mmol, 1.0 equiv) in diethyl ether. A bright yellow precipitate instantly formed. Water $(5 \mathrm{~mL})$ was added, which dissolved the precipitate and turned the solution light brown. The layers were separated and the aqueous phase was extracted three times with $5 \mathrm{~mL}$ portions of diethyl ether. The combined organic layers were dried over $\mathrm{MgSO}_{4}$ and concentrated under reduced pressure. NMR indicates a 6:1 ratio of desired product to starting material. Flash chromatography using a gradient elution (5:1 hexanes/ethyl acetate, then $2: 1$ hexanes/ethyl acetate) provided $95 \mathrm{mg}(43 \%)$ of 17 as a solid: m.p. $115-116^{\circ} \mathrm{C} .{ }^{\mathrm{H}} \mathrm{H}$ NMR $\left(500 \mathrm{MHz}, \mathrm{CDCl}_{3}\right): \delta 6.30(\mathrm{~d}, J=2.2 \mathrm{~Hz}, 1 \mathrm{H}), 5.98(\mathrm{~d}, 1.8$ $\mathrm{Hz}, 1 \mathrm{H}), 5.95(\mathrm{~s}, 1 \mathrm{H}), 2.92(\mathrm{~s}, 6 \mathrm{H}), 2.45(\mathrm{~s}, 3 \mathrm{H}) .{ }^{13} \mathrm{C} \mathrm{NMR}\left(125 \mathrm{MHz}, \mathrm{CDCl}_{3}\right): \delta$ 156.57, 156.40, $155.65,105.38,99.47,64.99,39.25,24.38 .{ }^{19} \mathrm{~F} \mathrm{NMR}\left(470 \mathrm{MHz}, \mathrm{CDCl}_{3}\right): \delta-143.8(\mathrm{dd}, J=22.1$ $\mathrm{Hz}, 8.4 \mathrm{~Hz}$ ), -155.9 (dd, $J=20.6 \mathrm{~Hz}, 20.6 \mathrm{~Hz}),-162.8$ (ddd, $J=22.1 \mathrm{~Hz}, 20.6 \mathrm{~Hz}, 8.4 \mathrm{~Hz}$ ). IR $3100-3500,1609,1505,1120,996 \mathrm{~cm}^{-1}$. TLC $\mathrm{R}_{\mathrm{f}}=0.47$ (ethyl acetate). Anal Calcd for $\mathrm{C}_{15} \mathrm{H}_{13} \mathrm{~F}_{5} \mathrm{~N}_{2} \mathrm{O}$ : C, 54.22; H, 3.94; N, 8.43. Found: C, 53.92; H, 3.85; N, 8.32.

\section{(4-Dimethylamino-6-methyl-pyridin-2-yl)-phenyl-methanol (18)}

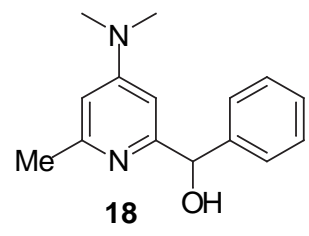

Bromobenzene $(77.0 \mu \mathrm{L}, 0.731 \mathrm{mmol}, 1.5$ equiv) was added via syringe to a flask containing magnesium turnings $(24.0 \mathrm{mg}, 0.974 \mathrm{mmol}, 2.0$ equiv) and diethyl ether $(0.5 \mathrm{~mL})$. The mixture was sonicated for 5 minutes at which point it appeared dark brown, and then diluted with diethyl ether $(2 \mathrm{~mL})$. The resulting Grignard reagent was then cannulated into a flask containing a solution of 4-(dimethylamino)-6-methyl-pyridine-2-carbaldehyde ( $80 \mathrm{mg}, 0.487 \mathrm{mmol}, 1.0$ equiv) in diethyl ether. A bright yellow precipitate instantly formed. Water $(5 \mathrm{~mL})$ was added, which dissolved the precipitate and turned the solution light brown. The layers were separated and the 
aqueous phase was extracted three times with $5 \mathrm{~mL}$ portions of diethyl ether. The combined organic layers were dried over $\mathrm{MgSO}_{4}$ and concentrated under reduced pressure. Flash chromatography using a gradient elution (5:1 hexanes/ethyl acetate, then 2:1 hexanes/ethyl acetate) provided $68 \mathrm{mg}(38 \%)$ of $\mathbf{1 8}$ as a solid: m.p. $141-142{ }^{\circ} \mathrm{C} .{ }^{1} \mathrm{H}$ NMR $\left(500 \mathrm{MHz}, \mathrm{CDCl}_{3}\right): \delta 7.38$ $(\mathrm{d}, J=7.5 \mathrm{~Hz}, 2 \mathrm{H}), 7.30(\mathrm{dd}, J=7.5 \mathrm{~Hz}, 7.5 \mathrm{~Hz}, 2 \mathrm{H}), 7.23(\mathrm{dd}, J=7.3 \mathrm{~Hz}, 7.3 \mathrm{~Hz}, 1 \mathrm{H}), 6.25(\mathrm{~d}, J$ $=2.0 \mathrm{~Hz}, 1 \mathrm{H}), 6.06(\mathrm{~d}, J=2.0 \mathrm{~Hz}, 1 \mathrm{H}), 5.90(\mathrm{br}, 1 \mathrm{H}), 5.55(\mathrm{~s}, 1 \mathrm{H}), 2.88(\mathrm{~s}, 6 \mathrm{H}), 2.45(\mathrm{~s}, 3 \mathrm{H}) .{ }^{13} \mathrm{C}$ NMR $\left(125 \mathrm{MHz}, \mathrm{CDCl}_{3}\right): \delta 160.07,156.28,155.37,144.20,128.31,127.37,127.07,104.81$, 100.92, 74.52, 39.18, 24.51. IR $3200-3500,1607,1546,1509,1217,1061 \mathrm{~cm}^{-1}$. TLC R $\mathrm{f}_{\mathrm{f}}=0.47$ (ethyl acetate).

\section{2-Pyrrolidin-1-ylmethyl-benzaldehyde.}

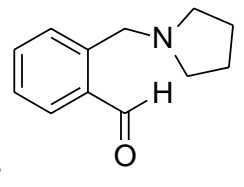

$t$-Butyllithium (1.7 $\mathrm{M}$ in ether, $7.66 \mathrm{~mL}, 13.0 \mathrm{mmol}, 2.3 \mathrm{equiv})$ was added via syringe to a $-78{ }^{\circ} \mathrm{C}$ solution of 1 -(2-bromo-benzyl)-pyrrolidine ${ }^{1}(1.36 \mathrm{~g}, 5.66 \mathrm{mmol}, 1.0$ equiv) in tetrahydrofuran $(50 \mathrm{~mL})$. The mixture was allowed to stir 1 hour at $-78{ }^{\circ} \mathrm{C}$ before $N, N$ dimethylformamide $(1.0 \mathrm{~mL}, 13.0 \mathrm{mmol}, 2.3$ equiv) was added via syringe. The mixture was allowed to warm to room temperature and stir overnight. Ethyl acetate $(50 \mathrm{~mL})$ and saturated sodium bicarbonate $(100 \mathrm{~mL})$ were added to the reaction mixture. The layers were separated and the aqueous layer was extracted with $50 \mathrm{~mL}$ ethyl acetate. The combined organic layers were dried over $\mathrm{MgSO}_{4}$ and concentrated under reduced pressure to a brown oil. Flash chromatography using a gradient elution (ethyl acetate, then 30:10:2 hexanes/ethyl acetate/triethylamine) afforded $925 \mathrm{mg}$ $(86 \%)$ of 2-pyrrolidin-1-ylmethyl-benzaldehyde as an orange oil. ${ }^{1} \mathrm{H}$ NMR $\left(500 \mathrm{MHz}, \mathrm{CDCl}_{3}\right): \delta$ $10.40(\mathrm{~s}, 1 \mathrm{H}), 7.80(\mathrm{dd}, J=7.5 \mathrm{~Hz}, 1.0 \mathrm{~Hz}, 1 \mathrm{H}), 7.45$ (ddd, $J=7.5 \mathrm{~Hz}, 7.5 \mathrm{~Hz}, 1.4 \mathrm{~Hz}, 1 \mathrm{H}), 7.34$ $(\mathrm{m}, 2 \mathrm{H}), 3.90(\mathrm{~s}, 2 \mathrm{H}), 2.45(\mathrm{~m}, 4 \mathrm{H}), 1.69(\mathrm{~m}, 4 \mathrm{H}) .{ }^{13} \mathrm{C} \mathrm{NMR}\left(125 \mathrm{MHz}, \mathrm{CDCl}_{3}\right): \delta 193.18$, $142.25,134.63,133.15,129.77,129.09,127.36,57.03,53.70,23.42$. TLC R $_{\mathrm{f}}=0.56(30: 10: 2$ hexanes /ethyl acetate/triethylamine). Anal Calcd for $\mathrm{C}_{12} \mathrm{H}_{15} \mathrm{NO}$ : C, 76.16; H, 7.99; N, 7.40. Found: C, 75.77; H, 7.63; N, 7.33.

\section{2,2,2-Trifluoro-1-(2-pyrrolidin-1-ylmethyl-phenyl)-ethanol (20)}

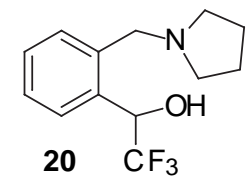

Tetrabutylammonium fluoride hydrate (catalytic) as a solution in tetrahydrofuran $(0.5 \mathrm{~mL})$ was added via cannula to a $0{ }^{\circ} \mathrm{C}$ solution of 2-pyrrolidin-1-ylmethyl-benzaldehyde $(50 \mathrm{mg}, 0.264$ mmol, 1.0 equiv) and trimethyl(trifluoromethyl)silane $(0.5 \mathrm{M}$ in tetrahydrofuran, $0.634 \mathrm{~mL}, 0.317$ mmol, 1.2 equiv) in tetrahydrofuran $(0.5 \mathrm{~mL})$. The solution was allowed to warm to room temperature and allowed to stir overnight. Hydrochloric acid $(6 \mathrm{M}, 88 \mu \mathrm{L}, 0.528 \mathrm{mmol}, 2.0$ equiv) was added to the mixture and stirred for 1 hour. Excess solid sodium carbonate was then added to neutralize the hydrochloric acid. The resulting mixture was diluted with ethyl acetate, filtered over $\mathrm{MgSO}_{4}$ and concentrated under reduced pressure to yield the hydrochloride salt as a white solid. The crude product was purified by flash chromatography (ethyl acetate, then 30:10:2 hexanes/ethyl acetate/triethylamine) to provide $53 \mathrm{mg}(77 \%)$ of 20 as a yellow oil. ${ }^{1} \mathrm{H}$ NMR $\left(500 \mathrm{MHz}, \mathrm{CDCl}_{3}\right)$ : $\delta 10.76(\mathrm{~s}, 1 \mathrm{H}), 7.28(\mathrm{~m}, 4 \mathrm{H}), 4.98\left(\mathrm{q}, J_{\mathrm{H}-\mathrm{F}}=8.1 \mathrm{~Hz}, 1 \mathrm{H}\right), 4.33(\mathrm{~d}, J=12.7 \mathrm{~Hz}, 1 \mathrm{H}), 3.25(\mathrm{~d}, J=$ $12.5 \mathrm{~Hz}, 1 \mathrm{H}), 2.57(\mathrm{~m}, 2 \mathrm{H}), 2.47(\mathrm{~m}, 2 \mathrm{H}), 1.76(\mathrm{~m}, 4 \mathrm{H}) .{ }^{13} \mathrm{C} \mathrm{NMR}\left(125 \mathrm{MHz}, \mathrm{CDCl}_{3}\right): \delta 137.45$, $135.03,131.40,129.03,128.09,125.68\left(\mathrm{q}, J_{\mathrm{C}-\mathrm{F}}=285 \mathrm{~Hz}\right), 75.96\left(\mathrm{q}, J_{\mathrm{C}-\mathrm{F}}=31.0 \mathrm{~Hz}\right), 59.74,52.87$, 23.24. ${ }^{19} \mathrm{~F}$ NMR $\left(376 \mathrm{MHz}, \mathrm{CDCl}_{3}\right): \delta-76.60\left(\mathrm{~d}, J_{\mathrm{H}-\mathrm{F}}=9.4 \mathrm{~Hz}\right)$. IR $3200-3400,1260,1162$, $1128 \mathrm{~cm}^{-1}$. TLC $\mathrm{R}_{\mathrm{f}}=0.49$, streak $\left(2: 1\right.$ hexanes/ethyl acetate). Anal Calcd for $\mathrm{C}_{13} \mathrm{H}_{16} \mathrm{~F}_{3} \mathrm{NO}: \mathrm{C}$, 60.22; H, 6.22; N, 5.40. Found: C, 60.26; H, 6.15; N, 5.41. 


\section{1-(2-Pyrrolidin-1-ylmethyl-phenyl)-ethanol (21)}

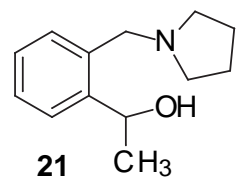

A solution of 2-pyrrolidin-1-ylmethyl-benzaldehyde (52 mg, $0.275 \mathrm{mmol}, 1.0$ equiv) in tetrahydrofuran $(3 \mathrm{~mL})$ was cooled to $0{ }^{\circ} \mathrm{C}$. Methyl magnesium bromide $(3.0 \mathrm{M}$ in diethyl ether, $110 \mu \mathrm{L}, 0.330 \mathrm{mmol}, 1.2$ equiv) was added to the solution via syringe and then the mixture was warmed to room temperature. After 5 minutes, an excess of water $(5 \mathrm{~mL})$ was added to quench the reaction. The reaction mixture was extracted with ethyl acetate. The organic phase was dried over $\mathrm{MgSO}_{4}$ and concentrated under reduced pressure. Flash chromatography (ethyl acetate) provided $29 \mathrm{mg}(51 \%)$ of 21 as a light yellow oil. ${ }^{1} \mathrm{H}$ NMR $\left(500 \mathrm{MHz}, \mathrm{CDCl}_{3}\right): \delta 7.87(\mathrm{~s}, 1 \mathrm{H}), 7.40(\mathrm{~d}, J$ $=7.7 \mathrm{~Hz}, 1 \mathrm{H}), 7.29(\mathrm{ddd}, J=7.5 \mathrm{~Hz}, 6.5 \mathrm{~Hz}, 2.4 \mathrm{~Hz}, 1 \mathrm{H}), 7.20(\mathrm{~m}, 2 \mathrm{H}), 5.01(\mathrm{q}, J=6.6 \mathrm{~Hz}, 1 \mathrm{H})$, $4.21(\mathrm{~d}, J=12.3 \mathrm{~Hz}, 1 \mathrm{H}), 3.27(\mathrm{~d}, J=12.1 \mathrm{~Hz}, 1 \mathrm{H}), 2.55(\mathrm{~m}, 2 \mathrm{H}), 2.45(\mathrm{~m}, 2 \mathrm{H}), 1.67(\mathrm{~m}, 4 \mathrm{H})$, $1.58(\mathrm{~d}, 6.6 \mathrm{~Hz}, 3 \mathrm{H}).) .{ }^{13} \mathrm{C}$ NMR $\left(125 \mathrm{MHz}, \mathrm{CDCl}_{3}\right): \delta 144.27,137.32,130.59,128.13,127.11$, 125.51, 66.18, 59.01, 53.22, 23.21, 20.26. TLC $\mathrm{R}_{\mathrm{f}}=0.29$ (30:10:2 hexanes /ethyl acetate/triethylamine). Anal Calcd for $\mathrm{C}_{13} \mathrm{H}_{19} \mathrm{~N}_{2} \mathrm{O}: \mathrm{C}, 76.06 ; \mathrm{H}, 9.33 ; \mathrm{N}, 6.82$. Found: C, 76.42; $\mathrm{H}$, $9.15 ; \mathrm{N}, 6.87$.

\section{1-[2-(2,2,2-Trifluoro-1-methoxy-ethyl)-benzyl]-pyrrolidine (22)}

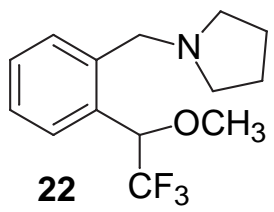

A solution 2,2,2-trifluoro-1-(2-pyrrolidin-1-ylmethyl-phenyl)-ethanol (101.8 mg, 0.393 mmol, 1.0 equiv) in tetrahydrofuran $(2 \mathrm{~mL})$ was added via cannula to a suspension of sodium hydride (50 mg, $1.96 \mathrm{mmol}, 5.0$ equiv) in tetrahydrofuran $(2 \mathrm{~mL})$ at room temperature. After 5 minutes, methyl iodide $(125 \mu \mathrm{L}, 1.96 \mathrm{mmol}, 5.0$ equiv) was added to the mixture via syringe. The reaction was allowed to stir 30 minutes. Brine was added dropwise to the mixture until the remaining sodium hydride was quenched (no visible activity observed) and then an additional $4 \mathrm{~mL}$ of brine was added. The mixture was then diluted with ethyl acetate and the layers were separated. The aqueous layer was extracted twice with ethyl acetate $(5 \mathrm{~mL})$ and the combined organic extracts were dried over $\mathrm{MgSO}_{4}$ and concentrated under reduced pressure. The crude oil was purified by flash chromatography (5:1 hexanes/ethyl acetate) to provide $49 \mathrm{mg}(46 \%)$ of 22 as a light yellow oil. ${ }^{1} \mathrm{H}$ NMR $\left(500 \mathrm{MHz}, \mathrm{CDCl}_{3}\right): \delta 7.59(\mathrm{~d}, J=7.5 \mathrm{~Hz}, 1 \mathrm{H}), 7.33(\mathrm{ddd}, J=7.3 \mathrm{~Hz}, 7.3 \mathrm{~Hz}, 1.6$ $\mathrm{Hz}, 1 \mathrm{H}), 7.29$ (ddd, $J=7.3 \mathrm{~Hz}, 7.3 \mathrm{~Hz}, 1.4 \mathrm{~Hz}, 1 \mathrm{H}), 7.23(\mathrm{~d}, J=7.3 \mathrm{~Hz}, 1 \mathrm{H}), 5.69\left(\mathrm{q}, J_{\mathrm{H}-\mathrm{F}}=87.0\right.$ $\mathrm{Hz}, 1 \mathrm{H}), 4.03(\mathrm{~d}, J=12.7,1 \mathrm{H}), 3.34(\mathrm{~s}, 3 \mathrm{H}), 3.22(\mathrm{~d}, J=12.9 \mathrm{~Hz}, 1 \mathrm{H}), 2.48(\mathrm{~m}, 2 \mathrm{H}), 2.37(\mathrm{~m}, 2 \mathrm{H})$, $1.72(\mathrm{~m}, 4 \mathrm{H}) .{ }^{13} \mathrm{C}$ NMR $\left(125 \mathrm{MHz} \mathrm{CDCl}_{3}\right): \delta 139.18,132.50,130.04,128.94,128.00,127.63$, $124.40,\left(\mathrm{q}, J_{\mathrm{C}-\mathrm{F}}=282 \mathrm{~Hz}\right), 75.38\left(\mathrm{q}, J_{\mathrm{C}-\mathrm{F}}=31.0 \mathrm{~Hz}\right), 58.68,57.35,53.83,23.61 .{ }^{19} \mathrm{~F} \mathrm{NMR}(376$ $\left.\mathrm{MHz}, \mathrm{CDCl}_{3}\right): \delta-76.56\left(\mathrm{~d}, J_{\mathrm{H}-\mathrm{F}}=9.0 \mathrm{~Hz}\right) . \quad \mathrm{IR} 1268,1168,1134,1087 \mathrm{~cm}^{-1}$. TLC $\mathrm{R}_{\mathrm{f}}=0.67(2: 1$ hexanes/ethyl acetate). Anal Calcd for $\mathrm{C}_{14} \mathrm{H}_{18} \mathrm{~F}_{3} \mathrm{NO}$ : C, 61.53; H, 6.64; N, 5.13. Found: C, 61.23; $\mathrm{H}, 6.51 ; \mathrm{N}, 5.08$.

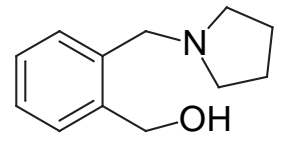

\section{(2-Pyrrolidin-1-ylmethyl-phenyl)-methanol (23)}

23

A solution of 2-pyrrolidin-1-ylmethyl-benzaldehyde (100 mg, $0.528 \mathrm{mmol}, 1.0$ equiv) in tetrahydrofuran $(1 \mathrm{~mL})$ was added via cannula to a suspension of lithium aluminum hydride $(20.0$ 
$\mathrm{mg}, 0.528 \mathrm{mmol}, 1.0$ equiv) in tetrahydrofuran $(4 \mathrm{~mL})$ at $0{ }^{\circ} \mathrm{C}$. The mixture was warmed to room temperature and allowed to stir two hours. Sodium hydroxide $(3.0 \mathrm{M})$ was added dropwise until a white precipitate formed. The mixture was diluted with ethyl acetate, filtered over $\mathrm{MgSO}_{4}$, and concentrated to a white solid. The crude product was purified by flash chromatography $(30: 10: 2$ hexanes/ethyl acetate/triethylamine) to provide $85 \mathrm{mg}(84 \%)$ of $\mathbf{2 3}$ as a pure white solid. ${ }^{1} \mathrm{H}$ NMR $\left(500 \mathrm{MHz}, \mathrm{CDCl}_{3}\right): \delta 7.26(\mathrm{~m}, 5 \mathrm{H}), 4.59(\mathrm{~s}, 2 \mathrm{H}), 3.70(\mathrm{~s}, 2 \mathrm{H}), 2.51(\mathrm{~m}, 4 \mathrm{H}), 1.73(\mathrm{~m}, 4 \mathrm{H}) .{ }^{13} \mathrm{C}$ NMR $\left(125 \mathrm{MHz}, \mathrm{CDCl}_{3}\right): \delta 141.42,137.98,130.05,129.75,128.20,127.69,64.92,59.25,53.33$, 23.21. IR $3300-3500,3100-3300,1022 \mathrm{~cm}^{-1}$. TLC $\mathrm{R}_{\mathrm{f}}=0.32$ (30:10:2 hexanes/ethyl acetate/triethylamine). Anal Calcd for $\mathrm{C}_{12} \mathrm{H}_{17} \mathrm{NO}$ : C, 75.36; H, 8.96; N, 7.32. Found: C, 75.52; $\mathrm{H}$, 8.97; N, 7.37.

[1] Prepared according to the procedure of Spector: Wilson, S.R.; Zucker, P.A.; Huang, R.; Spector, A. J. Am. Chem. Soc 1989, 111, 5936.

[2] Perrin, D.D. ; Armarego, W.L.F. Purification of Laboratory Chemicals Pergamon: Oxford, 1988. 